\title{
Evaluation of Skin Tumors by Magnetic Resonance Imaging
}

\author{
Moganty R. Rajeswari, Aklank Jain, Ashok Sharma, Dinesh Singh, \\ N. R. Jagannathan, Uma Sharma, and M. N. Degaonkar
}

Departments of Biochemistry (MRR, AJ, AS, DS) and Nuclear Magnetic Resonance (NRJ, US, MND), All India Institute of Medical Sciences, Ansari Nagar, New Delhi, India

SUMMARY: In vivo magnetic resonance imaging (MRI) is a powerful noninvasive technique in medical diagnosis; however, its application to analyze skin disorders is still at initial stages. To check whether MRI can be used as a noninvasive tool to analyze skin tumors, we carried out MRI of mice after treatment with benzo[a]pyrene (BP), a well known carcinogen. MRI was done on whole mice and was particularly focused on various layers and regions of interest of the skin: dermis, epidermis, and tumor. Initial MRIs of mice bearing skin tumors of $4,8,12$, and 16 weeks after inducing BP clearly revealed the appearance of tumor. The MRIs of tumor-bearing mice with 20-week-old tumor development showed invasion to adjacent internal anatomic structures. The MRI data were in good agreement with the extent of cellular atypia and neoplastic changes that are typical of squamous cell carcinoma as noticed from the histopathologic findings. Therefore, MRI seems to have the potential to evaluate the tumor invasions equally well as that of histopathology or other clinical findings. (Lab Invest 2003, 83:1279-1283).

$T$ he incidence of skin cancer in humans has been increasing for the past several years. A small fraction of these skin cancers are melanomas, which can readily spread to the lymphatics and blood stream and cause significant mortality. The vast majority of nonmelanoma skin cancers are basal cell carcinomas and squamous cell carcinomas (Grossman and Leffell, 1995). The latter represents one-third of nonmelanoma skin cancer and usually occurs in older individuals. Nonmelanoma skin cancer is generally caused by exposure to ionizing radiation, thermal radiation, aromatic hydrocarbons, and even by viruses like human papilloma virus. These nonmelanoma skin cancers need to be detected early and treated appropriately.

Although clinical examinations are important in all medical specialties, it is a critical element in the practice of dermatology. Lesions may be falsely considered benign, may not undergo biopsy, and therefore may not be subjected to the reference gold standard. Magnetic resonance imaging (MRI) so far has been extensively used for the diagnosis and evaluation of cancers associated with internal organs in the body. However, recently $\mathrm{MRI}$ is finding an interesting clinical application in evaluating skin tumors (Maurer et al, 1995). Because the thickness of the skin is small, MRI of the skin demands high resolution and high sensitivity. Recent developments

\section{DOI: 10.1097/01.LAB.0000088767.45565.27}

Received May 31, 2003.

Supported by Grant DST-SR/SO/AS-55/2003.

Address reprint requests to: Dr. Moganty R. Rajeswari, Department of Biochemistry, All India Institute of Medical Sciences, Ansari Nagar, New Delhi 110029, India.E-mail:mrraji@hotmail.com in surface coil technology and high field scanners have made MRI useful to study superficial skin structures at high resolution (Idy-Peretti et al, 1998; Richard et al, 1991).

Bond et al (1991) analyzed choroidal melanoma by MRI technique at various stages like pre- and postoperation. The application of MRI in dermatology can give a detailed picture of a tumor and its depth of invasion in relation to adjacent anatomic structures as well as delineate pathways of tumor invasion. Holzberg (1992) has reported the confirmation of the glomus tumor of the nail by MRI. In vivo MR spectroscopy has been used to differentiate the diseased skin from the normal skin (Kim et al, 1989; Song et al, 1997). The in vivo MRI in medical diagnosis has been reported to differentially evaluate malignant melanoma tumors (Maurer et al, 2000) and subcutaneous and pigmented skin of nodular and superficial spreading melanoma (Schwinghofer et al, 1989). Idy-Peretti et al (1998) have reported application of MRI for evaluating lymphodema in skin layers and subcutaneous tissue. An attempt has been made to evaluate unusual or aggressive tumors (eg, verrucous and sebaceous carcinoma) using MRI (Zemtsov et al, 1992a). Zemtsov et al (1992b) also suggest the possibility of MRI application in detecting the recurrence of skin cancer under skin flaps and grafts.

In the present study involving squamous cell carcinoma in mice, we used benzo[a]pyrene (BP), a potent chemical carcinogen and environmental genotoxin well known because of its negative effect on humans. We have also reported in animal models that BP increases the expression of onco-proteins, like high mobility group (HMG A1) DNA binding proteins (Rajeswari et al, 2001). It is also shown that 
benzo[a]pyrene-7,8-diol-9,10-eposide forms adducts with DNA (Bjelogrlic et al, 1994) and causes mutations in the Ha-ras oncogene (Ronaci et al, 1994). The mouse tumors help in the understanding of environ-

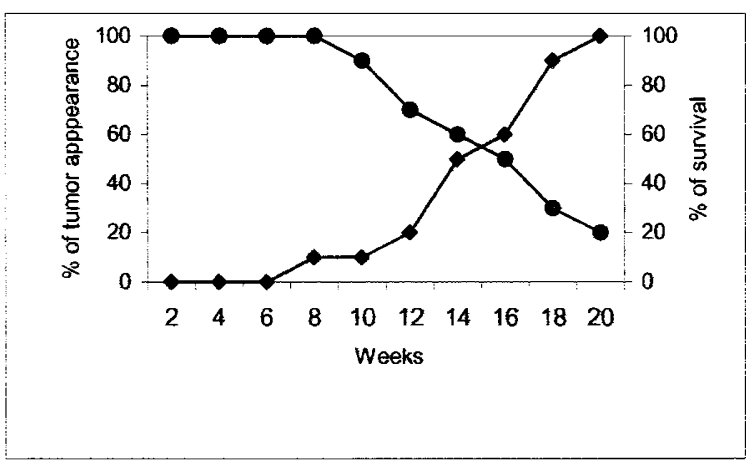

\section{Figure 1.}

Percentage of survival rate (-0-0) and skin tumor appearance (- $\mathbf{0}-\mathbf{0}-)$ in mice after topically applying benzo[a]pyrene (100 $\mu \mathrm{g}$ in $200 \mu$ l acetone) on the dorsal surface of mice.
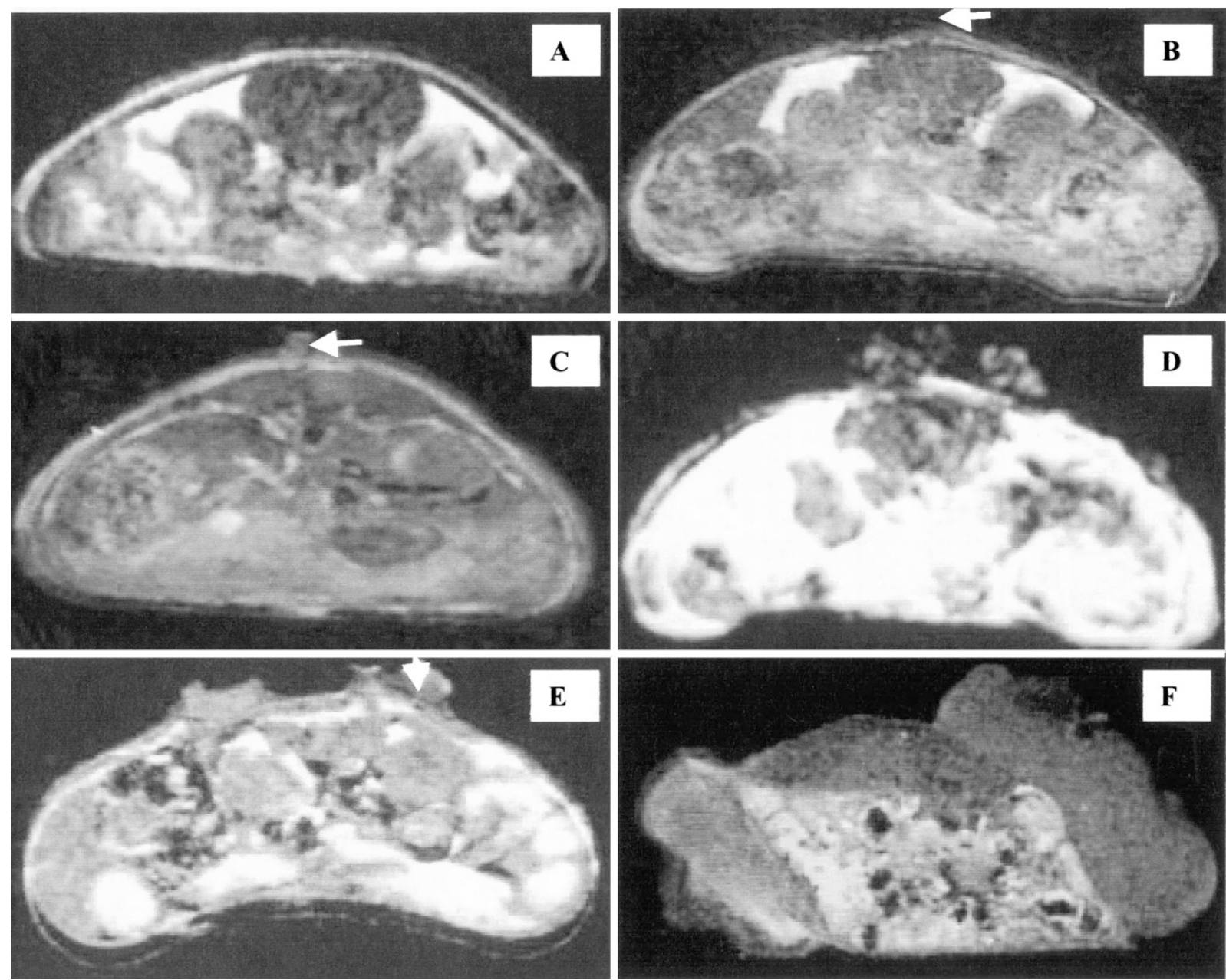

Figure 2.

Trans-axial multi spin-echo MRIs (echo-time $[T E]=15 \mathrm{msec}$, repetition time $[\mathrm{TR}]=600 \mathrm{msec}$ ) of control and tumor-bearing mice after inducing carcinogenesis with benzo[a]pyrene. (A) Control mice $(n=8)$; (B) tumor-bearing mice $(n=6)$ at 4 weeks, (C) 8 weeks, (D) 12 weeks, (E) 16 weeks, and (F) 20 weeks. A 72-mm diameter volume resonator was used as a transmitter/receiver coil. Field of view of $40 \times 40 \mathrm{~mm}$ and acquisition matrix size of $256 \times 256$ corresponding to the spatial resolution of $235 \times 235 \mu \mathrm{M}$ and with a 1-mm slice thickness. Tumors are indicated by arrows. 

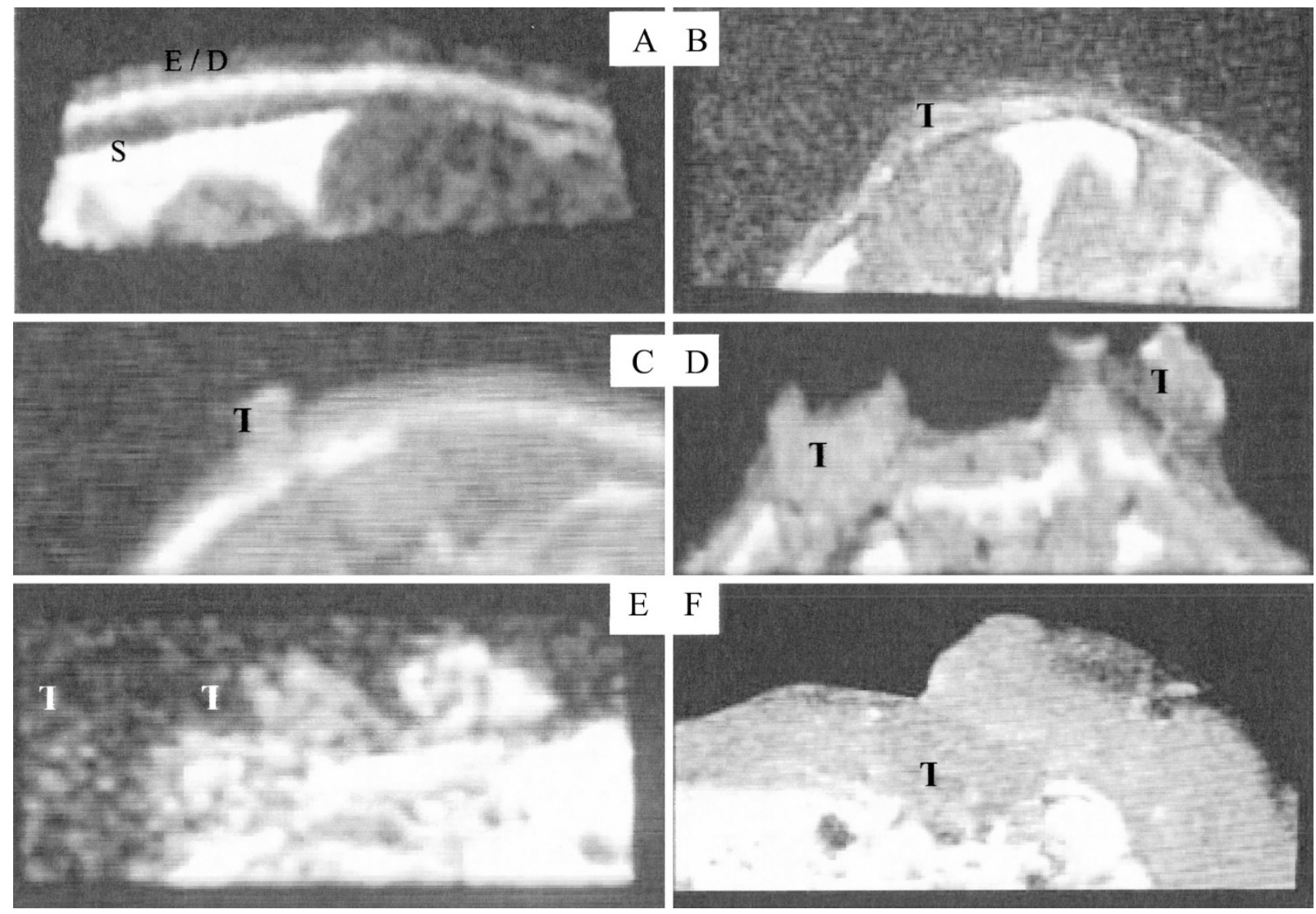

Figure 3.

A closer look at magnetic resonance images of the regions of interest (ROI) in Figure 2 (indicated by arrow). The image shown in $\mathrm{A}$ to $\mathrm{F}$ correspond to the ROI of A to $\mathrm{F}$ of Figure 2. $\mathrm{T}=$ tumor area, $\mathrm{E}=$ epidermis, $\mathrm{D}=$ dermis, and $\mathrm{S}=$ subcutis. The loss of dermis and epidermis interphase accompanied by the invasion of tumor area is clearly seen as the tumor advanced particularly after 12 weeks of carcinogenesis.

tumor responses, and by 16 weeks (Fig. 1), all of the mice were bearing tumors. The cumulative skin papilloma incidence of mice treated with BP is shown in Figure 1. The survival rate simultaneously decreased with time (Fig. 1), and at the end of 20 weeks, only $20 \%$ of the animals survived ( 2 of 10 ), although all of the mice developed full growth tumors. All of the data presented here are obtained with 8 number of mice for normal and 6 for tumor bearing mice.

$\mathrm{MRI}$ is more advantageous over other radiological techniques in analyzing some tissues. MRI gives better contrast (by about 25\%) than computed tomography. MRI is dependent on a number of parameters of protons, eg, density, $\mathrm{T} 1$ and $\mathrm{T} 2$ relaxation times, bulk motion, diffusion, magnetic susceptibility, and so forth. The cellular distribution of water with or without increase in global tissue water is different in cancerous tissue than that of normal skin. The increase in total proton density associated with changes in water structure alters free mobile protons in epidermis, dermis, and subcutaneous tissue. These changes reflect as contrast and brightness in MRIs. Quick pulse repetition times and short data collection times (echo times) are known as spin-echo times, and we have followed T1 weighted images in the present study. Because of the paramagnetic properties of skin tissue, the lesions and metastasis appear hypointense on T1.
The T1-weighted MRIs of transverse sections of mice were obtained by using a volume coil as shown in Figure 2, control $(A)$ and tumor ( $B$ to $F$ ). The resolution of these images is relatively poor compared with surface coil images. Initially, the MRls were obtained with a surface coil; however, during the course of the study, it was noticed that the lesion invaded deep into the inner organs (Fig. 2, B to F) hence a volume coil was used. This facilitated observing the extent of invasion of the lesion to the deep internal structures of the mice. Figure $2 \mathrm{~B}$ shows the MRI of a mouse taken after 4 weeks of BP exposure. The appearance of small lesions on the top layer of skin (epidermis) can be clearly seen in the images (indicated by arrows). The number of lesions on the skin and their size varied from mouse to mouse, and lesions at this time of study (4- to 8-week-old tumors) in most of the mice were confined to the epidermis (Fig. 2, B and C). The MRI of the same mice taken after 12 weeks of exposure to BP is shown in Figure 2C; the development of a number of small tumors is seen clearly. In addition, lesions present at 12 weeks onwards (Fig. 2, D to F) have grown bigger in size, and the image intensity (hypointensity) appears to be heterogeneous. Invasion of the lesion deep into the dermis layer of the skin is clearly seen in Figure 2, E and $\mathrm{F}$ as the tumor advanced to 16 to 20 weeks old. 
The images clearly indicate a considerable growth of the tumor in addition to the invasion into the internal anatomic structures.

To demonstrate the tumor invasion in the internal structure, it is necessary to view the regions of interest (ROI), ie, tumor regions, at a high resolution and sections of smaller thickness. The images given in Figure 3, A to F correspond to the ROI of the normal (A) and tumor (B to F) skin images represented by arrows in Figure 2, A to $F$, respectively. The epidermis and dermis junction is shown as "E" and "D" and subcutis as "S" in Figure 3A. The tumor-to-fat contrast is very considerably significant as the tumor shows considerably less signal intensity than fat in the T1weighted images (average about 20\%). As explained earlier malignant tumors appear as hypointense on T1-weighted images; the tumors are shown as " $T$ " in Figure 3, B to F. The changes seen in Figure 3, B to F are accompanied by the disappearance of dermalepidermal interphase and spreading of tumor deeper inside and are quite obvious. However, in the benign tumors (4 and 8 weeks old), the dermal-epidermal interphase was not totally lost (Fig. 3, B and C).

After the MRI the mouse was killed and histopathologic examination of skin biopsy was done. It must be mentioned here that the MRI and histology data are representative of the same mouse.

On light microscopic evaluation, in contrast to the control skin ( $n=8$ ) (Fig. 4A), skin biopsy of the tumor samples at 8 weeks revealed papillomatosis, focal parakeratosis, and acanthosis of the epidermis (Fig.
4B). The premalignant lesion at 4 weeks was also biopsied but did not reveal any papilloma (data not shown). The features of the tumor samples of 16 weeks (Fig. 4C) were typical of squamous cell papilloma. The biopsy of 20 weeks showed marked proliferation of squamous epithelium (Fig. 4D). The proliferation islands were invading deeper down with the presence of mitotic figures within squamous cells, and there was marked proliferation of the pilosebaceous units. Therefore, these results on MRI of skin tumor progression correlate well with the corresponding histopathologic evaluations.

From the present data, we suggest that MRI can be a used as a powerful, noninvasive imaging technique to determine the extent of tumor invasion and early detection of squamous cell carcinomas that are otherwise solely dependent on clinical and histopathologic examination. The role of MRI is constantly expanding and can progress as an important clinical technique for human skin cancers. In the future MRI may prove its use in dermatology in general.

\section{Materials and Methods}

Four-week-old female Swiss albino mice, obtained from the animal facility of the All India Institute of Medical Sciences, were maintained five per plastic cage. The animal maintenance practice was carried out in compliance with the guidelines of the institute's Animal Ethics Committee for the care and use of laboratory for experimental purpose (approval no.
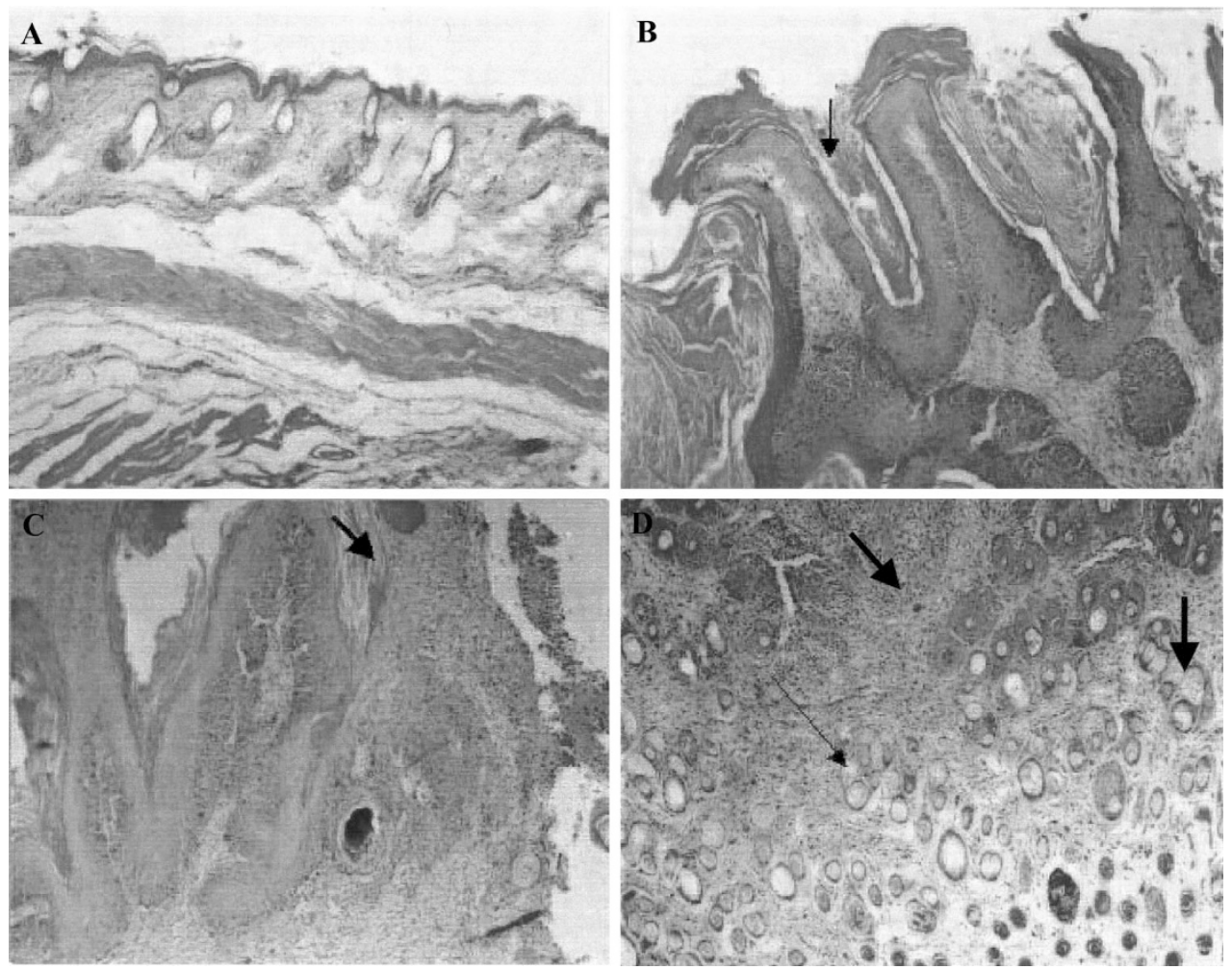

Figure 4.

Photo micrograph showing histopathology of mice skin control (A) and biopsy of tumor samples 8 weeks (B), 16 weeks (C), and 20 weeks (D) after induction of carcinogenesis. Hematoxylin-eosin staining, $\times 100 ; n=6$ in tumor samples and $n=8$ in normal samples. (See "Materials and Methods" for experimental details.) 
58/4/97 dated 5/10/99). BP was purchased from Sigma, and the purity was $>99 \%$. The animals were divided into three groups: normal (10 mice), controls (10 mice), and BP treated (30 mice). They were maintained in a temperature-controlled $\left(22 \pm 1^{\circ} \mathrm{C}\right)$ room, with food and water ad libitum. The normal mice did not received any treatment, the control mice were treated with only acetone solvent, and the BP-treated mice were given BP (100 $\mu \mathrm{g}$ in $200 \mu$ l acetone) topically on the dorsal surface once a week as described previously (Schwinghofer et al, 1989).

\section{MRI}

All animals were anesthetized with ip pentobarbital (30 to $40 \mathrm{mg} / \mathrm{kg}$ ) and scanned with MRI every 4 weeks to establish optimal imaging parameters. All of the MRIs were acquired using a Bruker BIOSPEC-4.5T animal MR scanner. The system is equipped with a maximum gradient strength of $210 \mathrm{mT} / \mathrm{m}$. A 72-mm diameter volume resonator was used as a transmitter/receiver coil. A multislice spin-echo RF pulse sequence was used, with a field of view of $40 \times 40 \mathrm{~mm}$ (acquisition matrix size of $256 \times 256$, corresponding to the spatial resolution of $235 \times 235 \mu \mathrm{M}$ and with a 1-mm slice thickness). Measurements of different ROI were obtained on the skin. The values were normalized with reference to the base line. The noise from the background was taken care of in determining the $\mathrm{S} / \mathrm{N}$ ratios.

\section{Histopathologic Examinations}

After performing the MRls, the mice were killed and the skin biopsy of each group was taken under aseptic conditions; histopathologic examinations were then done. The biopsy fragments were fixed in $10 \%$ neutral buffered formalin, and tissues were subjected to paraffin processing. Four- to $5-\mu \mathrm{M}$-thick sections were cut from the paraffin blocks and stained with hematoxylin-eosin for light microscopic evaluation.

\section{Acknowledgements}

We thank Dr. M. Ramam, Department of Dermatology, All India Institute of Medical Sciences, for his valuable comments and suggestions. We also thank the Department of Science and Technology for financial support (Grant DST-SR/SO/AS-55/2003).

\section{References}

Bjelogrlic NM, Makinen M, Stenback K, and Vahakangas $\mathrm{K}$ (1994). Benzo[a] pyrene-7, 8 diol-9, 10-epoxide DNA adducts and increased p53 in mouse skin. Carcinogenesis 15:771774.
Bond JB, Haik BG, Futosh M, and Gupta KL (1991). Magnetic resonance imaging of choroidal melanoma with and without gadolinium contrast enhancement. Ophthalmol 98:459-466.

Grossman D and Leffell DJ (1995). Squamous cell carcinoma and its precursors: Clinical biology and molecular basis. In: Chu, AC and Edelson RL editors. Malignant tumors of the skin. London: Arnold, Holder Head Line Group, 19-23.

Holzberg M (1992). Glomus tumor of the nail: A "red herring" clarified by magnetic resonance imaging. Arch Dermatol 128:160-162.

Idy-Peretti I, Bittoun J, Alliot FA, Richard SB, Querleux BG, and Cluzan RV (1998). Lymphedematous skin and subcutis: In vivo resolution magnetic resonance imaging evaluation. J Invest Dermatol 110:782-787.

Kim YH, Oroenberg EK, Faull KF, Ward-Jardetyky NG, and Jardetyky M (1989). 1H NMR Spectroscopy: An approach to evaluation of diseased skin in-vivo. J Invest Dermatol 92: 210-216.

Maurer J, Knolhmann FD, Schhms D, Garbe C, Vogl JJ, Bier I, and Felix R (1995). Role of high resolution magnetic resonance imaging for differentiating melanin containing skin tumors. J Invest Radiol 30:638-643.

Maurer J, Strauss A, Ebert W, Bauer H, and Felix R (2000). Contrast-enhanced high resolution magnetic resonance imaging of pigmented malignant melanoma Mn-TPPS4 and Gd-DTPA: Experimental results. Melanoma Res 10:40-46.

Mukhtar H, Mercurio G, and Agarwal R (1995). In: Murine carcinogenesis: Relevance to humans. Bonn: RC Press Inc., 3-8.

Rajeswari MR, Singh D, Jain A, and Ray R (2001). Elevated levels of high-mobility-group chromosomal proteins, HMGA1, in murine skin carcinoma. Cancer Lett 173:93-99.

Richard S, Querleux B, Bittom J, Idy-Peretti I, Jolivet O, Cermakona E, and Leueque JL (1991). In vivo proton relaxation time analysis of the main layers by magnetic resonance imaging. J Invest Dermatol 97:120-125.

Ronaci A, Scott G, El-Bayouamy K, and Stephen S (1994). Contrasting incidence of ras mutations in rat mammary and mouse skin tumors induced by anti-benzo [a] phenthrem-3, 4-diol, 1, 2-epoxide. Carcinogenesis 15:2113-2116.

Schwinghofer BW, Fruchwald FXI, Pohl-Markl H, Neuhold A, Wicke L, and Landrum WL (1989). MRI evaluation of pigmented skin tumors. J Invest Radiol 24:289-293.

Song HK, Wehrli Fw, and Ma J (1997). In vivo MR microscopy of the human skin. Magn Reson Med 37:185-191.

Zemtsov A, Koss W, Dixon L, Tyring S, and Rady P (1992a). Anal verrucous carcinoma associated with human papilloma virus type II: Magnetic resonance imaging and flow cytometry evaluation. Arch Dermatol 128:564-565.

Zemtsov A, Reed J, and Dixon L (1992b). Magnetic resonance imaging evaluation helps to delineate a recurrent skin cancer present under the skin flap. J Dermatol Surg Oncol 18:508-511. 